University at Albany, State University of New York

Scholars Archive

\title{
Analysis for Science Librarians of the 2013 Nobel Prize in Chemistry: The Work of Martin Karplus, Michael Levitt, and Arieh Warshel
}

Mary Van Ullen

University at Albany, State University of New York

Follow this and additional works at: https://scholarsarchive.library.albany.edu/ulib_fac_scholar

Part of the Library and Information Science Commons

\section{Recommended Citation}

Van Ullen, Mary, "Analysis for Science Librarians of the 2013 Nobel Prize in Chemistry: The Work of Martin Karplus, Michael Levitt, and Arieh Warshel" (2014). University Libraries Faculty Scholarship. 56.

https://scholarsarchive.library.albany.edu/ulib_fac_scholar/56

This Article is brought to you for free and open access by the University Libraries at Scholars Archive. It has been accepted for inclusion in University Libraries Faculty Scholarship by an authorized administrator of Scholars Archive. For more information, please contact scholarsarchive@albany.edu. 


\section{INTRODUCTION}

Over the past several decades, scientific understanding in the fields of chemistry and biochemistry has grown tremendously. For more than a century, molecules have been modeled using spherical representations of atoms, held together with sticks to represent chemical bonds. In more recent decades, the structures of large, complex molecules like proteins have been elucidated using instrumental analysis techniques including X-ray crystallography and Nuclear Magnetic Resonance spectroscopy.

While very useful in their own right, these techniques have limitations when trying to determine the function of complex chemical systems. Chemical reactions, such as photosynthesis or enzymatic catalysis, take place in a tiny fraction of a second. These interactions are too fleeting to capture with traditional experimental techniques. Further complicating the study of biological processes is that extremely large and complicated molecules are involved, yet the reactions of interest take place in reaction centers, a relatively small part of the molecule with only a few atoms involved (Fernholm 2013).

Scientists had used computer programs to gain greater understanding of chemical systems for some time. The static behavior even of very complex molecules could be modeled using software that applied classical Newtonian physics. Early in their careers, Dr. Arieh Warshel and Dr. Michael Levitt joined forces at the Weizmann Institute of Science in Israel to create such programs. One of the strengths of approaching molecular modeling from a Newtonian perspective is that it can be accomplished using relatively modest computer resources (Chang 2013). 
At about the same time, Dr. Martin Karplus and his colleagues at Harvard University were working on a different approach. They created software to simulate reaction mechanisms using quantum physics. While this approach gives the scientist detailed information about the behavior of the electrons and atoms involved in the chemical process being studied, quantum calculations require very substantial computing resources, and in the late 1960 s and into the 1970 s, available computer resources were extremely limited even at the most technologically advanced institutions. These types of calculations are too resource-intensive to be applied with sufficient accuracy to entire large and complicated biological systems (The Nobel Prize in Chemistry 2013 - Advanced Information).

The 2013 Nobel Prize in Chemistry was awarded for work involving the combination of both the classical Newtonian approach and the quantum physics approach in the multiscale computer modeling of complex systems.

\section{THE SCIENTISTS}

Martin Karplus

[INSERT Figure 1 Karplus Photo]

The younger of two sons, Martin Karplus was born in Austria in 1930. His parents took the family to the United States in 1938, to escape Nazi persecution after the Anschluss. The family moved to a suburb of Boston, and Karplus settled into the public school system. As a teenager, his father presented him with a microscope, and Karplus soon became fascinated with the study of nature and biology.

During Karplus' undergraduate studies at Harvard, he came to feel that in order to achieve a deeper understanding of biology, he needed to also study the physical sciences, and thus he entered the 
program in Chemistry and Physics (Karplus 2006). He began his Ph.D. at Caltech in biology, but decided to switch to chemistry. There he studied under Linus Pauling, himself the winner of both the Nobel Peace Prize and the Nobel Prize in Chemistry and one of the most influential and sometimes controversial scientific figures in the twentieth century. From Pauling, Karplus learned the importance of trusting his intuition and looking for logical solutions when approaching difficult problems (Ireland 2013). Karplus received his Ph.D. in 1955, and his dissertation was entitled, "A quantum-mechanical discussion of the bifluoride ion." He chose the bifluoride ion in part because more complex biologically active molecules were too difficult to study at that time using the relatively primitive and labor intensive methods available to do calculations in quantum mechanics (Karplus 2006).

While at Columbia and the University of Illinois at Urbana-Champaign early in his career, Karplus gained experience developing computer programs that modeled the quantum physics of reactions. At UrbanaChampaign, he developed mathematical models that were applicable to the powerful new tool of nuclear magnetic resonance (NMR). The relationship that came to be known as the Karplus equation gained much scientific interest and is now widely applied to the structural determination of proteins using NMR. Concerned that his work was being interpreted too widely, he published a short communication elaborating on the limitations of the Karplus equation and its proper usage (Karplus 1963). This became one of the most highly cited papers ever published in the Journal of the American Chemical Society (Dalton 2003).

In 1966, Martin Karplus accepted a position at Harvard University and has concurrently held a visiting professorship at the University of Strasbourg since 1995.

Michael Levitt

[INSERT Figure 2 Photo of Michael Levitt] 
Born and raised in Pretoria, South Africa, Michael Levitt received his B.Sc. in Physics from Kings College in the United Kingdom. Prior to starting work on his doctoral degree, he took a year to study at the Weizmann Institute, to learn about the work being done there on consistent force field theory, a method for modeling molecular properties using classical physics. At Weizmann, Levitt worked with Arieh Warshel, then a Ph.D. student, to write a computer program they named CFF. This program could be used to generate models of all types of molecules, both small and large. Levitt used the program to study the structure of two proteins, myoglobin and lysozyme, while Warshel applied it to determine the properties of small organic molecules.

Despite having access to CFF and excellent computing facilities, Levitt recalled a personal project to build a space-filling CPK physical model of transfer RNA, a subject of intensive study at the time. CPK models are a particular type of three-dimensional physical chemical representation named after Robert Corey, Linus Pauling and Walter Koltun, the chemists who established their use. The model of the macromolecule was so large that Levitt had to resort to lowering the structure out of the window of his cottage to complete it, and he measured the atomic distances with a plumb line on graph paper, a far cry from the elegance of his computer models (Levitt 2001).

Levitt received his Ph.D. from Cambridge in 1971, with a dissertation on "Conformational analysis of proteins," entirely based in computational biology. After spending several more years at Weismann and at Cambridge, he joined the faculty of the Department of Structural Biology, at Stanford University School of Medicine in 1987. (Levitt 2004)

Arieh Warshel

[INSERT Figure 3 Photo of Arieh Warshel] 
Arieh Warshel was born on a kibbutz in Israel's Beit She'an Valley in 1940. He served in the Israeli military in his youth, participating in both the Six Day and Yom Kippur Wars. He received his B.Sc. in Chemistry from Technion Israel Institute of Technology, and his Ph.D. in Chemical Physics from the Weizmann Institute of Science. In the 1960s, the Weizmann Institute was well-known for having some of the most robust computing capabilities available anywhere (Fiske 2013). Warshel's work during this time focused on computer modeling of the properties of small organic molecules (Levitt 2001).

After finishing his post-doctoral work at Harvard, he returned to Israel to take a faculty position at Weizmann. However, Warshel was not granted tenure at Weizmann, and in 1976 he joined the University of Southern California's Department of Chemistry, where he is currently Distinguished Professor of Chemistry.

Warshel's award of the Nobel Prize in Chemistry has generated discussion in the media about the difficult climate for scientists in Israel. Israel has suffered from the phenomenon of brain drain, with a high percentage of Israeli scientists leaving to work abroad, because of a lack of funding for research and education. Also problematic to young scientists is an insufficient infrastructure, along with the existence of relatively few tenured faculty posts (Sobelman 2013).

\section{THE COLLABORATIONS}

In 1970, Warshel, then a post-doctoral researcher, came to Karplus's lab at Harvard. The two collaborated using Warshel's experience with classical physics and Karplus's interest in quantum physics to develop a program that used quantum physics to perform calculations on free electrons, those electrons found in some molecules that are able to move readily between multiple atomic nuclei, but used classical physics to account for the remainder of the electrons and atomic nuclei. Although this work was important, it could only be applied to a certain class of molecules. 
During this period, Karplus's group was focused on understanding the properties of retinal, a molecule found in the eye that is essential to human vision. Retinal is a molecule with free electrons. When exposed to light, the free electrons absorb energy and the shape of the molecule changes. This reaction happens almost instantaneously, and like many biological reactions, it is much too rapid to be measured by traditional experimental techniques. After much work, Karplus and Warshel were able to use their approach of combining classical and quantum physics to model retinal (Fernholm 2013).

After Warshel left Harvard, he resumed his collaboration with Michael Levitt at the Medical Research Council Laboratory in Cambridge, England. The two worked on modeling enzymatic reactions, an area of particular interest to Warshel. Enzymes are essential to biochemical processes, and because they are typically large protein molecules with a specific reactive site where the chemical process of interest is rapidly facilitated, they are prime subjects for the application of multiscale molecular modeling (Fernholm 2013). In 1976, they published a study of an enzymatic process that was ground-breaking because it used a model that could be used for any type of molecule (Warshel and Levitt 1976).

Additional work by Warshel and Levitt produced the discovery that the modeling of parts of the molecule away from the reactive sites can be further simplified by grouping some atoms in their calculations (Fernholm 2013). They also found that in the study of enzymes it was important to allow for the effects of the surrounding media, especially water (Chang 2013).

\section{THE PUBLICATIONS}

Unsurprisingly, all three of the 2013 Laureates are highly productive researchers, but they experienced skepticism from their colleagues for the purely computational nature of their work. Martin Karplus recalled submitting a manuscript predicting some structural characteristics of retinal, along with his collaborator Barry Honig, to the journal Nature. The paper was rejected because it lacked experimental 
data confirming the calculated conformation. Karplus noted, "This was my first experience with Nature and with the difficulty of publishing theoretical results related to biology, particularly in high impact journals. The problem is almost as prevalent today as it was then, i.e., if the theory agrees with the experiment it is not interesting because the result is already known, whereas if one is making a prediction, then it is not publishable because there is no evidence that the prediction is correct." (Karplus 2006, 29) Still, Karplus persisted, and Nature eventually published the work. Arieh Warshel noted resistance from the community of experimental researchers to the newly emerging field of computational chemistry for anything but findings which substantiated experimental results. They tended to reject any conclusions from his work that could not be measured directly by experimental methods. He stated, "The last thing people want is that you will come and explain their systems. I never succeeded to convince anyone. I just made them angry." (Chang 2013)

The results of Karplus's Citation Report in Web of Science's citation databases run on December 22, 2013 are represented in Figure 4. A total of 927 items were found with Martin Karplus listed as an author. His earliest work listed in the Web of Science search was published in 1952 and concerned a study of arctic bird populations, reflecting his lifelong interest in biology. Dr. Karplus continues to publish in the field. His period of maximum scholarly output began in the mid-1980s and lasted well over twenty years.

[INSERT Figure 4]

One indication of the significance of Dr. Karplus's work is that his publications have been cited 102,297 times, averaging 110.3 citations per item. Karplus, born in 1930 , is the oldest of the three laureates honored in 2013. His h-index, as found in the Web of Science, is 151. A high h-index is a reflection of a prolific author with publications of sustained interest and above-average impact, and is dependent on the author being mature in their publishing career (Bornmann 2007). While the typical h-index of Nobel 
Prize winners in the sciences would be expected to be high, the metric for Karplus is impressive even compared to the dozen Nobel laureates in Chemistry from 2006 to 2011, who had an average h-index of 74.6. (Antonucci-Durgan 2012). Only Robert Lefkowitz, one of the 2012 winners, had a higher h-index at the time of his award (Lutz 2013).

Born in 1947, Michael Levitt is the youngest of the three computational chemists. He has a much smaller number of publications (162) to his credit than do Karplus or Warshel, but his publications have each been cited an average of 133 times. The bulk of Levitt's publications have appeared from 1989 to the present (Figure 5). His h-index, at a value of 70, is lower than that of Warshel and Karplus but given his younger age it is likely to increase over time.

\section{[INSERT Figure 5]}

Arieh Warshel's publication history began in 1968, and according to the Web of Science, he has 450 published works that have been cited an average of 76.4 times. While he has published steadily during his career, the number of publications climbed in the latter half of the 1990s, and he continues to publish a significant number of items each year, as is shown in Figure 6. His h-index is an impressive 100.

[INSERT Figure 6]

Tables 1, 2 and 3 provide information about the top publication outlets for Karplus, Levitt and Warshel, respectively. In addition to the traditional journal literature, both Karplus and Warshel have a long history of participation in the American Chemical Society Meetings, as is evidenced by the large number of entries appearing in the Abstracts of Papers of the American Chemical Society. Not surprisingly, there is a great deal of overlap in the titles which have published their research, particularly for Karplus and Warshel who have a more extensive body of published work. 
[INSERT Tables 1, 2 and 3]

Also included in the three tables are the associated 5-Year Impact Factors taken from the online 2012 Journal Citation Reports (JCR). This metric is the average number of times that articles from a journal published in the past five years have been cited in the given Journal Citation Report year. It is determined by dividing the number of citations in the Journal Citation Reports year by the total number of articles published in the five prior years. This information is included to give some indication of the relative importance in recent years of the journals in which the laureates have heavily published.

Citation practices show considerable variation between disciplines, and thus what is an impressive impact factor in one discipline might be quite different in another. The Journal Citation Reports database can be accessed to find category metrics by subject. Because the discoveries of the laureates incorporate facets of biochemistry, physical chemistry, computer science and other disciplines, journals in which the 2013 Nobel prize winners have tended to publish do not fit neatly into JCR journal categories. Table 4 shows a list of four of the most relevant JCR subject categories, along with the median Impact Factors for each category. By comparison, most of the titles in which Karplus, Warshel and Levitt have published much of their work have substantially higher Impact Factors than the JCR median Impact Factors for the categories, an indication that their work has been well accepted by the important journals in their field. This includes some of the top interdisciplinary journals in the sciences, such as Nature and The Proceedings of the National Academy of Sciences of the United States of America (PNAS).

\section{[INSERT Table 4]}

The term used by the Royal Swedish Academy of Science in awarding this prize, "Multiscale Models," is not unique, and is found applied in many other fields. It is not a useful search term in the literature. To 
gain a basic understanding of the impact of this type of computational analysis on the field of chemistry over time, a search was performed in the SciFinder database on the three authors, and was analyzed by index term. The resulting lists of index terms appearing in the publications of the authors were scanned to identify frequently appearing terms that were most appropriately descriptive of the work. Two terms, "molecular dynamics" and "conformation" appeared very frequently as index terms for all three sets of publications, and together are descriptive of the concepts honored by the award.

To see how these terms appeared in the literature over time, an additional SciFinder search was performed looking for the appearance of both terms in journal articles indexed in the CAPLUS database. A total of 24,444 journal articles were found. Figure 7 breaks out this total by year, beginning in 1970 , when a single article met the criteria. In the early 1990s, appearance of the terms increased sharply, with a steady annual increase. In 2012, 1,980 such articles appeared in the chemical literature, one indication of the growth of the field.

[INSERT Figure 7]

\section{CONCLUSION}

The work associated with the 2013 Nobel Prize in Chemistry has been focused on insight into how complex biological systems work, and without it, scientists would have a much less sophisticated understanding of processes including the mechanisms of enzymatic reactions, the folding and packing of proteins, and the prediction of biomolecular conformations. It is possible that multiscale computer modeling will advance understanding of the biological sciences much further. As Michael Levitt noted, "Computers were made for biology: biology would never have advanced as far as it did without the dramatic increase in computer power and availability. One day we would like to be able to simulate complicated biological processes, perhaps even going from the genomic sequence to a full simulation of the organism's phenotype." (Levitt 2001, 393) 
The foundational work of Karplus, Warshel and Levitt has much broader scientific implications. The types of computer modeling techniques available now can be used, for example, in the development of new drugs, in the discovery of solid catalysts and in the engineering of new materials. Because computer technology is one of the rare situations where costs are decreasing at the same time that capability is growing, the importance of computational methods in science and engineering will only continue to grow (Jacoby 2013). As Arieh Warshel observed, "The past 25 years have taught us that we cannot predict how the field of molecular modeling will mature." (Warshel 2002, 393)

\section{ACKNOWLEDGMENT}

The author would like to thank Thomson Reuters for providing publication and citation data.

\section{REFERENCES}

Antonucci-Durgan, D. 2012. Science Librarians Analysis of the 2011 Nobel Prize in Chemistry: The Work of Dan Schechtman. Science \& Technology Libraries 31 (1): 1-11.

Bornmann, L. and H. Daniel. 2007. What do we know about the $\mathrm{h}$ index? Journal of the American Society for Information Science \& Technology 58 (9): 1381-5.

Chang, Kenneth. 2013. Without test tubes, 3 win Nobel in Chemistry. New York Times. Available at http://www.nytimes.com/2013/10/10/science/three-researchers-win-nobel-prize-inchemistry.html .

Dalton, L. 2003. Karplus equation: Theoretical calculation links NMR coupling constant to molecular geometry. Chemical and Engineering News 81 (51): 37. 
Fernholm, A. 2013. Taking the experiment to cyberspace. The Nobel Prize in Chemistry 2013. Available at http://www.nobelprize.org/nobel_prizes/chemistry/laureates/2013/popularchemistryprize2013.pdf.

Fiske, G. 2013. Three Jewish professors- two of them Israeli- share 2013 Nobel Prize in Chemistry. Times of Israel. Available at http://www.timesofisrael.com/israeli-prof-arieh-warshel-shares-2013nobel-prize-in-chemistry/.

Ireland, C. 2013. Harvard professor wins Nobel in chemistry. HARVARDgazette. Available at http://news.harvard.edu/gazette/story/2013/10/harvard-professor-wins-nobel-in-chemistry/.

Jacoby, M. 2013. Chemistry by the numbers: From data collection and analysis to quantum calculations, computers have revolutionized chemistry. Chemical \& Engineering News 91 (36): 51-5.

Karplus, M. 1963. Vicinal proton coupling in nuclear magnetic resonance. Journal of the American Chemical Society 85 (18): 2870-1.

Karplus, M. 2006. Spinach on the ceiling: A theoretical chemist's return to biology. Annual Review of Biophysics \& Biomolecular Structure 35 (1): 1-47.

Levitt, M. 2001. The birth of computational structural biology. Nature Structural Biology 8 (5): 392-3.

Levitt, M. 2004. Curriculum vitae. Available at http://csb.stanford.edu/levitt/.

Lutz, E. 2013. Science Librarians Analysis of the 2012 Nobel Prize in Chemistry: The Work of Robert Lefkowitz and Brian Kobilka. Science \& Technology Libraries (1): 19-29. 
The Official Web Site of the Nobel Prize. 2013. The Nobel prize in chemistry 2013 - Advanced Information. The Nobel Prize in Chemistry 2013. Available at http://www.nobelprize.org/nobel_prizes/chemistry/laureates/2013/advanced.html.

Sobelman, B. 2013. Nobel Prize in chemistry puts spotlight on Israel's brain drain. Los Angeles Times. Available at http://www.latimes.com/world/worldnow/la-fg-wn-nobel-israel20131010,0,4762305.story.

Warshel, A. 2002. Molecular dynamics simulations of biological reactions. Accounts of Chemical Research 35(6): 385-95.

Warshel, A. and M. Levitt. 1976. Theoretical studies of enzymic reactions: Dielectric, electrostatic and steric stabilization of the carbonium ion in the reaction of lysozyme. Journal of Molecular Biology 103(2), 227-249. 